\title{
Magnetic Resonance Imaging Demonstration of Fascicular Inflammation in Sixth Nerve Palsy as the Presenting Symptom in Multiple Sclerosis
}

\author{
Kristina Szabo Rolf Kern Martin Griebe Hansjoerg Baezner Michael G. Hennerici \\ Achim Gass
}

Department of Neurology, Universitätsklinikum Mannheim, University of Heidelberg, Mannheim, Germany

Dear Sir,

The abducens nerve is a small anatomical structure and the most commonly affected nerve in ocular motor nerve palsies $[1,2]$. Recent progress in magnetic resonance imaging (MRI) sequence technology has made it possible not only to visualize pathology in the pontine parenchymal portion of the sixth nerve but also in the cisternal segment $[3,4]$.

\section{Case 1}

A 32-year-old woman presented with a 3-day history of horizontal diplopia, which worsened on right gaze. She recalled having a diffuse new type of headache of 4hour duration on the evening prior to symptom onset. She was referred to an ophthalmology department and a brain MRI was ordered, which was reported as normal. Subsequently, she was referred to the Neurology Department. Her past medical and neurological history was negative. On neurological examination, there was an isolated sixth nerve palsy on the right. In addition, she reported slight dysesthesia of the left calf, without objective sensory loss on examination.

Cerebrospinal fluid (CSF) analysis revealed a slightly elevated cell count $(8 / \mu \mathrm{l})$ with normal protein $(393 \mathrm{mg} / \mathrm{l})$ and glu- cose (58 mg/dl) levels, intrathecal IgG and IgM production, and positive oligoclonal bands - therefore, a second MRI was performed assuming a possible inflammatory sixth nerve lesion. High resolution MRI of the sixth nerve demonstrated a normal cisternal segment but a lesion of the fascicular part of the sixth nerve on the right (fig. 1), without any further brain or spinal cord abnormalities at that point.

Visually evoked potentials showed a delayed and abnormal potential on the right, indicating possible asymptomatic slight optic nerve involvement. Results of auditory, somatosensory and motorevoked potentials were normal. Extensive laboratory investigations of serum and CSF failed to show evidence of an alternative etiology, and thus, the diagnosis of possible multiple sclerosis (MS) was made. The patient was treated with intravenous methylprednisolone $500 \mathrm{mg} /$ day for 3 days showing fast and marked improvement of symptoms, and complete resolution of diplopia and weakness of abduction subsequently. Follow-up MRI at 3 and 6 months demonstrated multiple new periventricular brain lesions fulfilling McDonald criteria [5] of MS.

\section{Case 2}

A 58-year-old man complained about slight double vision for 2 days and reported similar visual disturbances 4 weeks earlier that had resolved spontaneously. His past medical history was unremarkable, except for mild untreated hypertension. Neurological examination showed sixth nerve palsy on the left without any definite further deficits. Surprisingly, MRI performed on the third day after symptom onset revealed $\mathrm{T}_{1}$-hypointense and $\mathrm{T}_{2}$-hyperintense lesions in the pons as well as in the periventricular white matter and in the corpus callosum, and several juxtacortical lesions. Ring-like contrast enhancement was demonstrated in one of the supratentorial lesions (fig. 2). On high resolution images, there was again a $\mathrm{T}_{2}$-hyperintense lesion along the fascicular course of the sixth nerve on the left, and slight contrast enhancement following the course of the nerve to the pontomedullary sulcus (fig. 2). The cisternal segment of the sixth nerve was normal, and diffusion-weighted images did not show corresponding lesions - as one initial differential diagnosis had been a possible ischemic pontine lesion.

Analysis of CSF showed a normal cell count $(1 / \mu \mathrm{l})$ with normal protein $(347$

\section{KARGER}

Fax +4161306 1234 E-Mail karger@karger.ch www.karger.com
Dr. Kristina Szabo

Department of Neurology, Universitätsklinikum Mannheim

Theodor-Kutzer-Ufer 1-3, DE-68167 Mannheim (Germany)

Tel. +49621 383 3202, Fax +49621383 3807

E-Mail szabo@neuro.ma.uni-heidelberg.de 

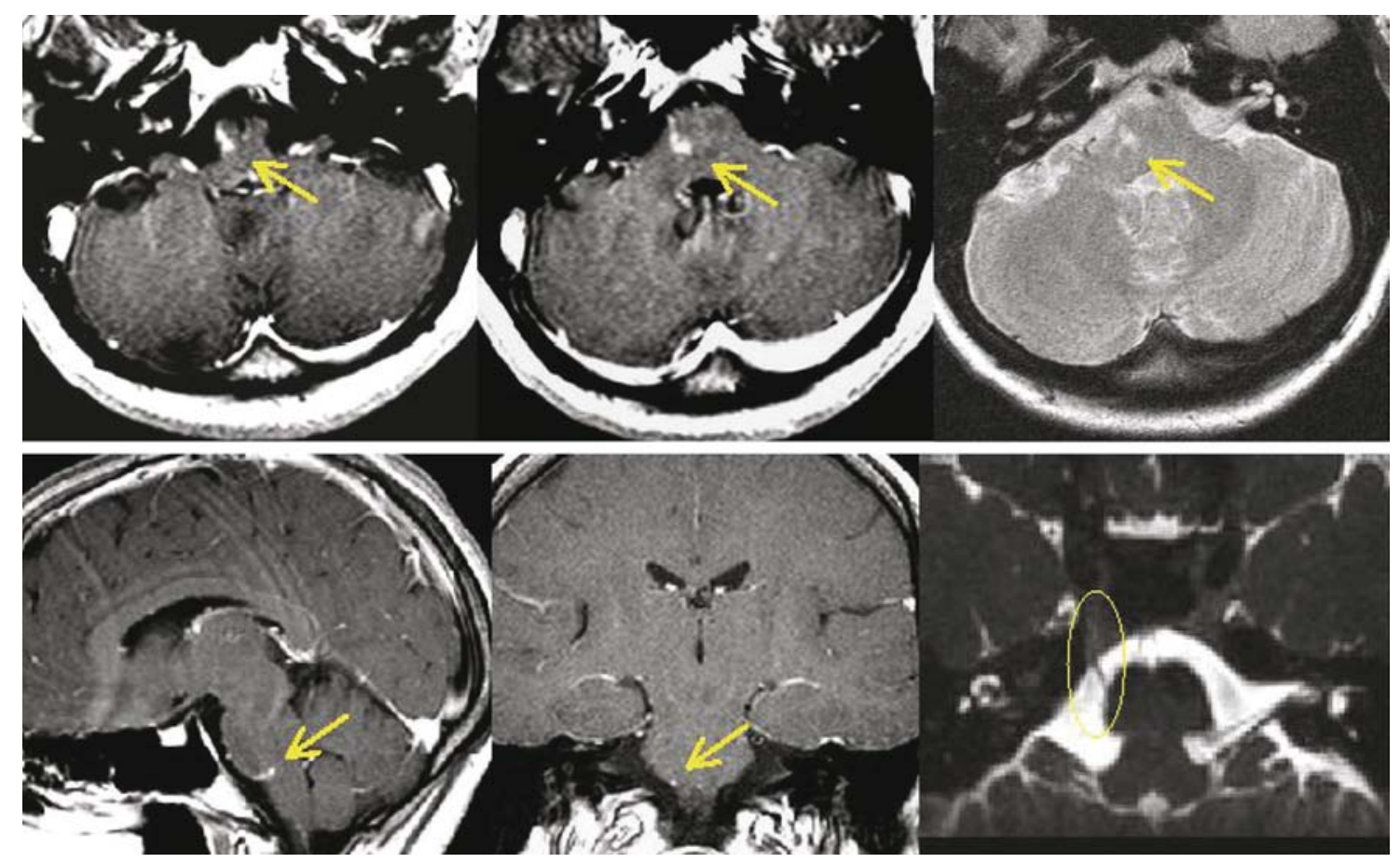

Fig. 1. Postcontrast $T_{1}$-weighted transverse (top row, left and center), sagittal (bottom row, left) and coronal (bottom row, center) MRI 5 days after onset of right sixth nerve palsy. An enhancing lesion along the intrapontine course of the right sixth nerve fascicle and a dot-like enhancement at the exit of the nerve in the pontomedullary sulcus is clearly seen (arrows). The transverse $\mathrm{T}_{2}$-weighted image (top row, right) demonstrates a corresponding hyperintense lesion, while the oblique axial reconstructed heavily $\mathrm{T}_{2}$-weighted image (bottom row, right) shows the normal cisternal portion of the nerve.

$\mathrm{mg} / \mathrm{l})$ and glucose $(61 \mathrm{mg} / \mathrm{dl})$ levels, but intrathecal IgG production and positive oligoclonal bands. Visually and auditory evoked potentials were normal, while somatosensory and motor-evoked potentials showed pathologic latency/conduction times bilaterally. The patient was treated with intravenous methylprednisolone 500 $\mathrm{mg} /$ day for 5 days, which was followed by immediate improvement, and he was discharged with minimal weakness of abduction. The diagnosis of possible MS was made after further tests had revealed no other inflammatory or infectious etiology. MS was also confirmed clinically when the patient presented with an acute sensory relapse 7 months after the sixth nerve palsy.

\section{Discussion}

In both patients, MRI illustrated fascicular inflammation in sixth nerve palsy as the presenting symptom of MS. The sixth nerve is the most commonly affected cranial nerve in oculomotor palsy with a broad range of differential diagnoses. In a recent retrospective study of 137 new cases of sixth nerve palsy, $7 \%$ were associated with MS [6]. Clinically isolated syndromes often present as brain stem symptoms, while sixth nerve palsy is an infrequent presentation. A retrospective review of 600 patients found a prevalence of $0.5 \%$ of an isolated abducens palsy as presenting sign of MS after exclusion of alternative pathologies [7].

Although the abducens nerve is an exquisitely small structure, modern MRI can demonstrate large parts of it reliably and has all options to demonstrate or rule out various pathologies. Different portions of the sixth nerve pathways can be visualized by different MRI sequences with high sensitivity: high resolution $\mathrm{T}_{2}$ - and $\mathrm{T}_{1}$-weighted MRI after contrast agent administration can demonstrate pathology in the nuclear region, fascicular course and exit zone of the sixth nerve in the pontomedul- lary sulcus, while MR angiographic source images and high resolution, strongly $\mathrm{T}_{2}$ weighted images visualize the cisternal path and can rule out vascular compression [3]. Diffusion-weighted MRI is often useful to demonstrate cytotoxic edema of ischemia as opposed to inflammatory edema in MS [8].

This report illustrates the fascicular inflammation of the sixth nerve and shows the accuracy of MRI to reliably identify such lesions. High resolution MRI frequently demonstrates fascicular involvement of the trigeminal nerve in MS, as a cause of trigeminal neuralgia or sensory disturbances [9]. Interestingly, the lesions in those cases also show a tendency to cover or follow the pontine anatomical course of the nerve fibers. This may well be regarded a sign with some specificity, as the main differential diagnosis of pontine lesions on MRI, namely ischemia of a ramus ad pontem, is usually wedge shaped and clearly different in this regard. 


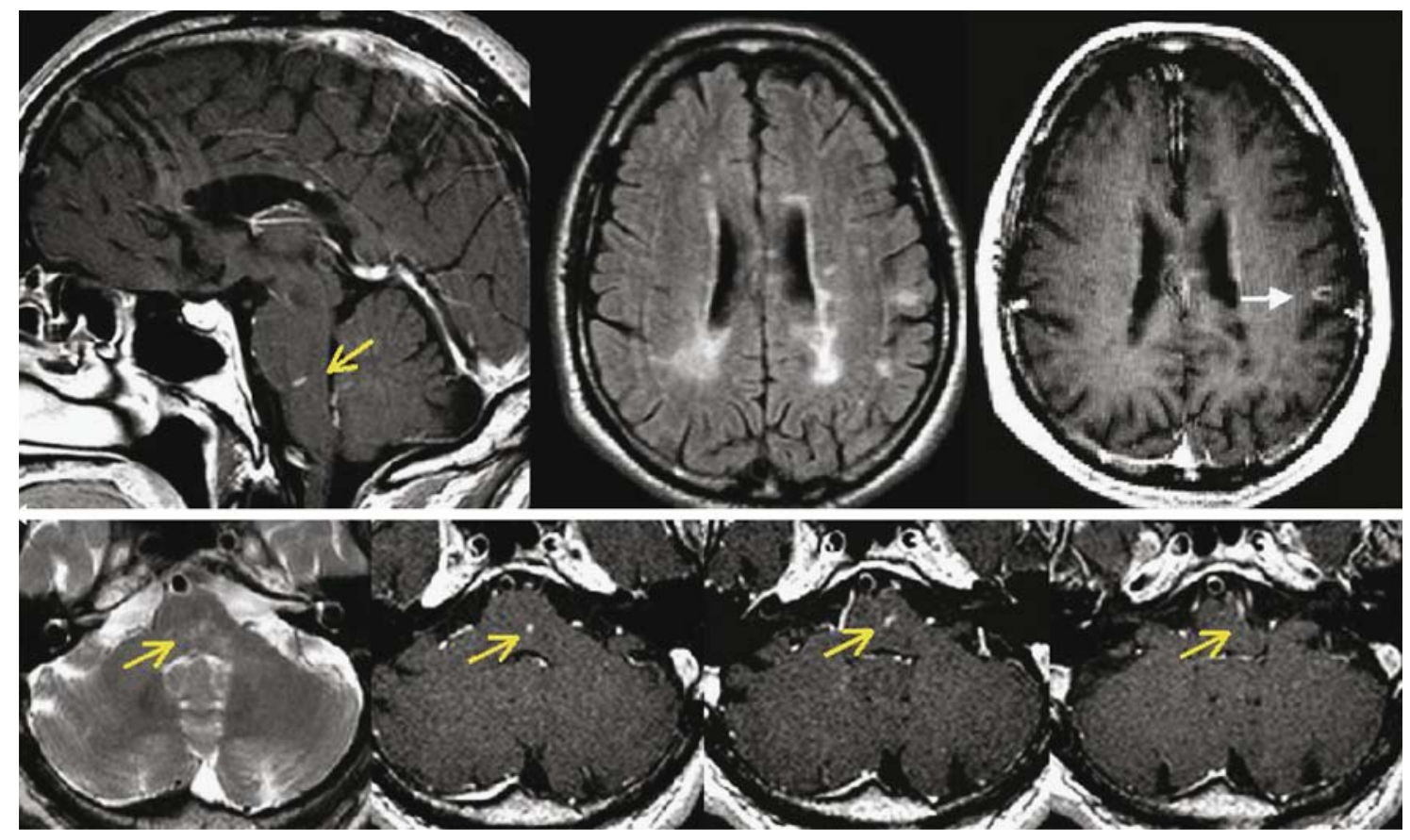

Fig. 2. Postcontrast $T_{1}$-weighted sagittal (top row, left) and transverse (bottom row, all except left) MRI on the third day of symptoms showing enhancing lesion along the intrapontine course of the left abducens nerve fascicle and at the exit of the nerve in the pontomedullary sulcus (arrows; top row, left, and bottom row, all). The transverse $\mathrm{T}_{2}$-weighted image (bottom row, left) shows corresponding diffuse hyperintense signal change. Supratentorial hyperintense periventricular lesions on FLAIR images (top row, center) with a ring-enhancing juxtacortical lesion on postcontrast $\mathrm{T}_{1}$-weighted image (arrow; top row, right) pointed to subsequently diagnosed MS

\section{References}

1 Rucker CW: The causes of paralysis of the third, fourth and sixth cranial nerves. Am J Ophthalmol 1966;61:1293-1298.

-2 Richards BW, Jones FR Jr, Younge BR: Causes and prognosis in 4,278 cases of paralysis of the oculomotor, trochlear, and abducens cranial nerves. Am J Ophthalmol 1992;113: 489-496

-3 Yousry I, Camelio S, Wiesmann M, Schmid UD, Moriggl B, Bruckmann H, Yousry TA: Detailed magnetic resonance imaging anatomy of the cisternal segment of the abducent nerve: Dorello's canal and neurovascular relationships and landmarks. J Neurosurg 1999;91:276-283.
4 Bendszus M, Beck A, Koltzenburg M, Vince $\mathrm{GH}$, Brechtelsbauer D, Littan T, Urbach $\mathrm{H}$, Solymosi L: MRI in isolated sixth nerve palsies. Neuroradiology 2001;43:742-745.

-5 Polman CH, Reingold SC, Edan G, Filippi M, Hartung HP, Kappos L, Lublin FD, Metz LM, McFarland HF, O'Connor PW, SandbergWollheim M, Thompson AJ, Weinshenker BG, Wolinsky JS: Diagnostic criteria for multiple sclerosis: 2005 revisions to the 'McDonald Criteria'. Ann Neurol 2005;58: 840-846.

-6 Patel SV, Mutyala S, Leske DA, Hodge DO, Holmes JM: Incidence, associations, and evaluation of sixth nerve palsy using a population-based method. Ophthalmology 2004; 111:369-375.
7 Barr D, Kupersmith MJ, Turbin R, Bose S, Roth R: Isolated sixth nerve palsy: an uncommon presenting sign of multiple sclerosis. J Neurol 2000;247:701-704.

8 Paik JW, Kang SY, Sohn YH: Isolated abducens nerve palsy due to anterolateral pontine infarction. Eur Neurol 2004;52:254-256.

-9 Gass A, Kitchen N, MacManus DG, Moseley IF, Hennerici MG, Miller DH: Trigeminal neuralgia in patients with multiple sclerosis: lesion localization with magnetic resonance imaging. Neurology 1997;49:1142-1144. 